\title{
Robust Array Calibration using Time Delays with Application to Ultrasound Tomography
}

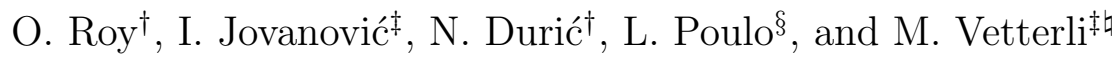 \\ † Karmanos Cancer Institute, \\ 4100 John R, \\ Detroit, MI 48201, USA \\ ‡ Audiovisual Communications Laboratory, \\ Ecole Polytechnique Fédérale de Lausanne, \\ CH-1015 Switzerland \\ $\S$ Analogic Corporation, \\ 8 Centennial Drive, \\ Peabody, MA 01960, USA \\ $\natural^{\natural}$ Department of Electrical Engineering and Computer Sciences, \\ University of California, \\ Berkeley, CA 94720, USA.
}

\begin{abstract}
Accurate calibration is a requirement of many array signal processing techniques. We investigate the calibration of a transducer array using time delays. We derive a strategy based on the mean square error criterion and discuss how time delays that are not available can be interpolated from existing ones. The proposed method is made robust to noise and model mismatch by means of a novel iterative technique for distance matrix denoising. The convergence of the method is proved. Finally, the accuracy of the proposed calibration algorithm is assessed both in simulated scenarios and using experimental data obtained from an ultrasound scanner designed for breast cancer detection.
\end{abstract}

Keywords: Array calibration, distance matrix, iterative denoising, transducer, ultrasound tomography.

Further author information: (Send correspondence to O. Roy)

O. Roy - email: royo@karmanos.org, phone: +1 3135768246

I. Jovanović - email: ivana.jovanovic@epfl.ch, phone: +41216931271

N. Durić - email: duric@karmanos.org, phone: +1 3135768706

L. Poulo - email: lpoulo@analogic.com, phone: +1 9783264000

M. Vetterli - email: martin.vetterli@epfl.ch, phone: +41 216935698

Medical Imaging 2011: Ultrasonic Imaging, Tomography, and Therapy, edited by Jan D'hooge, Marvin M. Doyley, Proc. of SPIE Vol. 7968, 796806 - (c) 2011 SPIE · CCC code: 1605-7422/11/\$18 · doi: 10.1117/12.877459 


\section{INTRODUCTION}

Arrays of transducers are found in a large number of practical applications. These include the design of multimicrophone systems for noise reduction, ${ }^{1}$ the deployment of a network of sensors to sample physical fields in space and time, ${ }^{2}$ or the building a ring of ultrasound transducers for tomographic imaging of breast tissues. ${ }^{3}$ Many of the signal processing algorithms designed for such applications rely on an accurate calibration of the experimental setup in order to achieve peak theoretical performance. However, calibration in the presence of noise and various types of model mismatch between the assumed and actual setup is a challenging problem which has been addressed by a large number of research studies (see, e.g., Refs. 4,5). Deviation from the assumed model occurs due to various reasons, including electronic noise, non-ideal transducer characteristics, inhomogeneities in the propagation medium and inaccurate measurements. A calibration method should thus be robust to provide a high level of accuracy even in these non-ideal scenarios.

We propose a transducer array calibration technique for systems requiring time delay measurements. These time delays can be computed from the signals transmitted and received between pairs of transducers using a variety of time delay estimation methods (see, e.g., Refs. 6,7). The propagation of these signals is typically governed by a wave equation as in acoustics, electromagnetics and fluid dynamics. The transducers have unknown positions and unknown processing delays. Moreover, the propagation medium is assumed to be homogeneous with an unknown propagation speed. The goal of the calibration method is to estimate the above unknowns in a robust and computationally efficient manner. To solve this estimation problem, we present an approach based on a mean square error criterion. In the case where time delay measurements between some pairs of transducers are unavailable, we interpolate the missing data using some prior knowledge about the distances between the transducers. The matrix formed by all pairwise distances, referred to as a distance matrix, enjoys a number of key properties. In particular, its rank is equal to 4 regardless of the number of transducers. This fact was used in Ref. 8 to design a provably accurate matrix completion method in a sensor network scenario.

Array calibration has been addressed by a large number of research studies (for a review see, e.g., Ref. 9). The first class of methods is of stochastic nature, that is, the noise corrupting the measurements is modeled as a random quantity with a given distribution (usually Gaussian). This is the approach adopted, for example, in Ref. 10 and references therein. The second class of methods, to which our own algorithm belongs, employs a deterministic formulation where the noise term is not made explicit. In this scenario, robustness to noise can be obtained by means of various optimization strategies. In particular, strategies based on a mean square error criterion similar to ours have been considered by many authors (see, e.g., Refs. 4,8). In this context, the novelty of our work compared to existing studies essentially resides in an iterative technique, based on the signal enhancement method exposed in Ref. 11, that uses properties of a distance matrix to denoise the measurements as a means to provide a robust estimate of the unknown calibration parameters. Another denoising strategy for distance matrices was proposed in Ref. 12. It is based on a minimization of an heuristic approximation of the rank of a matrix. The accuracy of the proposed method is first assessed using numerical simulations. We then consider the calibration of an ultrasound transducer array used in a scanner prototype for breast cancer detection. ${ }^{3}$ In this scenario, good calibration plays an important role in producing high resolution artifact-free images of breast tissues using, for example, travel time tomography ${ }^{13}$ or waveform tomography. ${ }^{14}$ We show that the proposed scheme can estimate the unknown parameters with a high accuracy and good consistency.

The outline of the paper is as follows. In Section 2, we establish the notation and state the calibration problem. The proposed algorithm is presented in Section 3. More specifically, an estimation technique based on the mean square error criterion is derived in Section 3.1 and the considered interpolation method is described in Section 3.2. We then describe the proposed iterative denoising procedure in Section 4. Results are presented in Section 5. The accuracy of the proposed calibration method is first assessed using numerical simulations in Section 5.1. Section 5.2 then considers the application of the method to an existing ultrasound transducer array used in a breast cancer detection modality. Conclusions are given in Section 6 .

\section{PROBLEM STATEMENT}

We consider an array of $n$ transducers with emit and receive capabilities. Let us denote by $\mathbf{x}_{i}$ the position of transducer $i(i=0,1, \ldots, n-1)$. For simplicity of exposure, the positions are taken in a two dimensional plane. 


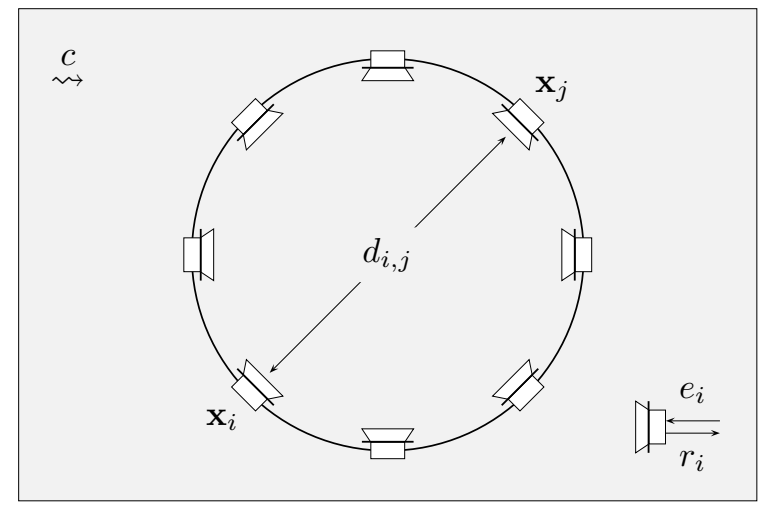

Figure 1. Array of $n$ transducers. Transducer $i$ has positions $\mathbf{x}_{i}$, emission delay $e_{i}$ and reception delay $r_{i}$. The distance between a pair of transducers is denoted by $d_{i, j}(i, j=0,1, \ldots, n-1)$. The environment is homogeneous with speed of propagation $c$.

Extension to the three dimensional scenario is straightforward. Each transducer is assumed to have unknown but fixed emission and reception delays denoted by $e_{i}$ and $r_{i}$, respectively. The emission delay refers to the electronic delay between the time we excite the system using a pulse, and the actual propagation of that pulse through the medium. The reception delay is defined similarly. We denote by $m_{i, j}$ the time delay measured between the probing signal sent to transmitter $i$ and the signal recorded at receiver $j$. This delay can be decomposed as

$$
m_{i, j}=t_{i, j}+e_{i}+r_{j}
$$

where $t_{i, j}$ denotes the actual propagation time between transducers $i$ and $j$, hereafter referred to as time of flight. The signals are assumed to propagate in an homogeneous environment with unknown propagation speed $c$. The time of flight $t_{i, j}$ thus satisfies

$$
d_{i, j}=c t_{i, j}
$$

where $d_{i, j}=\left\|\mathbf{x}_{i}-\mathbf{x}_{j}\right\|$ is the Euclidian distance between transducers $i$ and $j$. The setup is depicted in Figure 1.

Our goal is to estimate the positions $\mathbf{x}_{i}$, the delays $e_{i}$ and $r_{i}$, as well as the speed of propagation $c$ using the measured time delays $m_{i, j}$. In practice, however, we only have access to a noisy version of $m_{i, j}$, denoted by $\hat{m}_{i, j}$. This estimate can be obtained using various time delay estimation methods (see, e.g., Refs. 6,7). The origin of the noise is manifold but includes electronic noise, non-ideal sensor characteristics and inhomogeneities in the propagation medium. When the signal-to-noise ratio is too weak to provide a relevant estimate, the measurement $\hat{m}_{i, j}$ can even be missing.

To formalize our problem, let us define the time of flight matrix $\mathbf{T}$, the distance matrix $\mathbf{D}$ and the measurement matrix $\mathbf{M}$ such that $(\mathbf{T})_{i, j}=t_{i, j},(\mathbf{D})_{i, j}=d_{i, j}$ and $(\mathbf{M})_{i, j}=m_{i, j}$, respectively. Observe that the distance matrix only depends on the position of the sensors $\mathbf{x}_{i}$, and that $\mathbf{D}=c \mathbf{T}$. We also define the delay vectors $\mathbf{e}$ and $\mathbf{r}$ whose $i$ th components are respectively given by $e_{i}$ and $r_{i}$. Estimated or noisy versions of these quantities are denoted with a hat (e.g., $\hat{\mathbf{r}})$. Our goal is to find the parameters $\hat{c}, \hat{\mathbf{e}}, \hat{\mathbf{r}}$ and $\hat{\mathbf{x}}_{i}$ that best fit the measurements in a mean square sense. More precisely, we would like to solve the following optimization problem

$$
\min _{c, \mathbf{e}, \mathbf{r}, \mathbf{x}_{i}}\|\mathbf{M}-\hat{\mathbf{M}}\|^{2}=\min _{c, \mathbf{e}, \mathbf{r}, \mathbf{x}_{i}}\left\|c^{-1} \mathbf{D}+\mathbf{e} \mathbf{1}_{n}^{T}+\mathbf{1}_{n} \mathbf{r}^{T}-\hat{\mathbf{M}}\right\|^{2},
$$

where $\|\mathbf{M}\|=\sqrt{\operatorname{tr}\left(\mathbf{M}^{T} \mathbf{M}\right)}$ is the Frobenius norm, $\mathbf{1}_{n}$ denotes the all-one vector of size $n$, and the dependency of the matrix $\mathbf{D}$ on the positions $\mathbf{x}_{i}$ is implicit. The equality in (3) follows directly from (1) and (2).

At this point of the derivation, two important observations need to be made. First, the above optimization problem has an inherent scaling ambiguity, that is, the optimal values for $c$ and the distance matrix $\mathbf{D}$ (computed from the positions $\mathbf{x}_{i}$ ) can only be determined up to a positive scaling factor. Second, it is non-convex and, as 
such, does not admit a simple and efficient solution. To address these issues, we propose to first estimate the sound speed and the transducer delays using a fairly good initial estimate of the transducer's positions. We then compute an updated distance matrix from which new positions can be estimated. Let us now explain our calibration algorithm in more details.

\section{CALIBRATION ALGORITHM}

\subsection{Mean Square Optimality}

Let us first rewrite the optimization (3) in a more convenient way. We have that

$$
\begin{aligned}
\left\|c^{-1} \mathbf{D}+\mathbf{e} \mathbf{1}_{n}^{T}+\mathbf{1}_{n} \mathbf{r}^{T}-\hat{\mathbf{M}}\right\|^{2} & =\left\|\operatorname{vec}\left(c^{-1} \mathbf{D}\right)+\operatorname{vec}\left(\mathbf{e} \mathbf{1}_{n}^{T}\right)+\operatorname{vec}\left(\mathbf{1}_{n} \mathbf{r}^{T}\right)-\operatorname{vec}(\hat{\mathbf{M}})\right\|^{2} \\
& =\left\|c^{-1} \mathbf{d}+\mathbf{K}_{e} \mathbf{e}+\mathbf{K}_{r} \mathbf{r}-\hat{\mathbf{m}}\right\|^{2},
\end{aligned}
$$

where $\mathbf{d}=\operatorname{vec}(\mathbf{D})$ and $\hat{\mathbf{m}}=\operatorname{vec}(\hat{\mathbf{M}})$ are vectors obtained by stacking the columns of $\mathbf{D}$ and $\hat{\mathbf{M}}$, respectively. The matrices $\mathbf{K}_{e}$ and $\mathbf{K}_{r}$ are given by $\mathbf{K}_{e}=\mathbf{1}_{n} \otimes \mathbf{I}_{n}$ and $\mathbf{K}_{r}=\mathbf{I}_{n} \otimes \mathbf{1}_{n}$, where $\otimes$ denotes the Kronecker product and $\mathbf{I}_{n}$ the identity matrix of size $n \times n$. Defining the matrix $\mathbf{K}$ and the vector $\mathbf{p}$ as $\mathbf{K}=\left[\begin{array}{lll}\mathbf{I}_{n^{2}} & \mathbf{K}_{e} & \mathbf{K}_{r}\end{array}\right]$ and $\mathbf{p}^{T}=\left[\begin{array}{lll}c^{-1} \mathbf{d}^{T} & \mathbf{e}^{T} & \mathbf{r}^{T}\end{array}\right]$, the optimization problem (3) can be expressed as

$$
\min _{c, \mathbf{e}, \mathbf{r}, \mathbf{x}_{i}}\|\mathbf{K} \mathbf{p}-\hat{\mathbf{m}}\|^{2} .
$$

To address the aforementioned scaling ambiguity, one can estimate the propagation speed as a separate measurement, and then estimate the positions $\hat{\mathbf{X}}^{T}=\left[\hat{\mathbf{x}}_{0}, \hat{\mathbf{x}}_{1}, \ldots, \hat{\mathbf{x}}_{n-1}\right]$ and delay vectors $\hat{\mathbf{e}}$ and $\hat{\mathbf{r}}$ using (5). This strategy requires a good estimate of the propagation speed. For example, in the experimental setup discussed in Section 5.2, the speed of propagation can be inferred from the temperature measurement obtained by a built-in thermometer. However, this approach only provides a single local estimate of the quantity of interest and is thus sensitive to inhomogeneities in the propagation medium.

The alternative approach that we are going to consider for the rest of the discussion is to start from an initial estimate $\mathbf{d}_{0}$ of the distance vector. The main advantage is that an estimation error in the distance between a pair of transducers is averaged over an order of $n^{2}$ measurements. Using $\mathbf{d}_{0}$, the optimization problem (5) reduces to

$$
\min _{c, \mathbf{e}, \mathbf{r}}\|\mathbf{K} \mathbf{p}-\hat{\mathbf{m}}\|^{2}
$$

where the matrix $\mathbf{K}$ and the vector $\mathbf{p}$ are now defined as

$$
\mathbf{K}=\left[\begin{array}{lll}
\mathbf{d}_{0} & \mathbf{K}_{e} & \mathbf{K}_{r}
\end{array}\right] \text { and } \mathbf{p}^{T}=\left[\begin{array}{lll}
1 / c & \mathbf{e}^{T} & \mathbf{r}^{T}
\end{array}\right] .
$$

The estimated sound speed $\hat{c}$ and delay vectors $\hat{\mathbf{e}}$ and $\hat{\mathbf{r}}$ can be retrieved from $\hat{\mathbf{p}}$ obtained by orthogonal projection as $\hat{\mathbf{p}}=\mathbf{K}^{\dagger} \hat{\mathbf{m}}$, where $\mathbf{K}^{\dagger}$ is the Moore-Penrose pseudoinverse.

In order to increase the accuracy of the initial estimate of the distance vector, the estimated sound speed $\hat{c}$ can be used to solve the original optimization problem (5). However, the cost function in (5) is non-convex and typically contains a large number of local minima such that the search of a solution is computationally intensive and very dependant on the chosen starting point. A comparison of this approach with our technique will be provided in Section 5.1. Instead, our approach amounts to simply compute the new distance vector estimate as

$$
\hat{\mathbf{d}}=\hat{c}\left(\hat{\mathbf{m}}-\mathbf{K}_{e} \hat{\mathbf{e}}-\mathbf{K}_{r} \hat{\mathbf{r}}\right) .
$$

Positions estimates are then computed using the well-known multi dimensional scaling (MDS) localization algorithm described in Algorithm 1 (see, e.g., Refs. 8,15). Note that the estimated distance vector $\hat{\mathbf{d}}$ is invariant with respect to translation, rotation and symmetry of the array. The positions derived from $\hat{\mathbf{d}}$ are thus relative, but absolute positioning can easily be obtained by specifying a few references (e.g., the center of the array, the position of a reference transducer). 


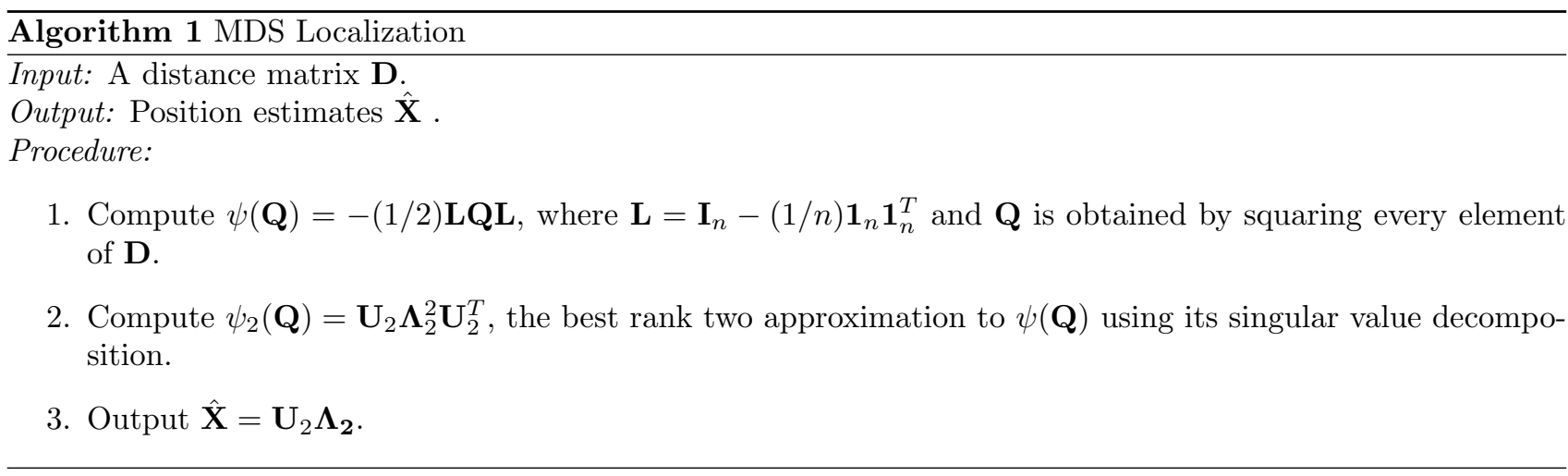

\subsection{Interpolation of Missing Measurements}

The above method requires that the entire measurement matrix is available for the estimation of the positions. In many practical scenarios, however, some of these measurements are missing or are simply too noisy to be considered as valid.

The strategy that we adopt in this work is to interpolate the missing data based on the initial distance estimates. More precisely, let us consider the diagonal weight matrix $\mathbf{W}$ of size $n^{2} \times n^{2}$ defined as $(\mathbf{W})_{i, j}=1$, if $i=j$ and $(\hat{\mathbf{m}})_{i}$ is available, and zero otherwise. Our approach consists in estimating the parameters $\hat{c}, \hat{\mathbf{e}}, \hat{\mathbf{r}}$ using the non-zero values of the vector $\mathbf{W} \hat{\mathbf{m}}$ and the corresponding initial distance estimates. This amounts to replacing the minimization (6) by the weighted mean square optimization problem

$$
\min _{c, \mathbf{e}, \mathbf{r}}\|\mathbf{W}(\mathbf{K} \mathbf{p}-\hat{\mathbf{m}})\|^{2} .
$$

The missing measurements are then estimated from the initial distance estimates $\mathbf{d}_{0}$ using the relationships $(1)$ and (2). Finally, the distances are updated using (8).

\section{DISTANCE MATRIX DENOISING}

In the absence of noise, a distance matrix $\mathbf{D}$, or equivalently the time of flight matrix $\mathbf{T}=c^{-1} \mathbf{D}$, possesses a specific structure owing to the nature of the measurements. More specifically, let us consider the matrix $\mathbf{Q}$ obtained by squaring every element of a distance matrix $\mathbf{D}$. It has the following key properties: (i) it is symmetric, (ii) it has zero diagonal elements, (iii) it has non-negative elements, and (iv) it is of rank at most 4.

The first three properties are obvious. The fourth one can be easily shown (see, e.g., Ref. 8) and suggests that, while a distance matrix contains $n^{2}$ entries, the number of degrees of freedom in designing such a matrix is no greater than $8 \mathrm{n}$. Its content is thus highly redundant since $n^{2}$ is typically much greater than $8 \mathrm{n}$. Note that, in the general multidimensional scenario, the rank is given by $d+2$, where $d$ is the dimension of the problem (here $d=2$ ).

In the presence of noise, however, the matrix $\mathbf{Q}$ does not generally satisfy all of the above properties. The idea is to enforce these properties as a means to denoise the underlying distance matrix. This is achieved by successively applying a number of mappings on the noisy data. The first mapping $\varphi_{1}$ enforces the symmetry of the matrix and is defined as $\varphi_{1}(\mathbf{Q})=\left(\mathbf{Q}+\mathbf{Q}^{T}\right) / 2$. The second mapping $\varphi_{2}$ ensures that the matrix has zero diagonal elements by setting $\left(\varphi_{2}(\mathbf{Q})\right)_{i, j}=(\mathbf{Q})_{i, j}$ if $i \neq j$, and zero otherwise. The third mapping $\varphi_{3}$ forces the matrix to have non-negative entries by setting its negative elements to zero, that is, $\left(\varphi_{3}(\mathbf{Q})\right)_{i, j}=(\mathbf{Q})_{i, j}$ if $(\mathbf{Q})_{i, j} \geqslant 0$, and zero otherwise. The fourth mapping implements the low rank condition by only retaining the four largest singular values. It is defined as $\varphi_{4}(\mathbf{Q})=\mathbf{U}_{4} \boldsymbol{\Lambda}_{4} \mathbf{V}_{4}^{T}$, that is, the best rank 4 approximation of $\mathbf{Q}$ using its singular value decomposition. The proposed denoising procedure is summarized in Algorithm 2. It amounts to successively applying the above mappings on the original noisy data. Note that the symmetry condition imposed by the function $\varphi_{1}$ is not violated by any of the subsequent mappings. It thus only needs to be applied at the 
Algorithm 2 Distance Matrix Denoising

Input: A noisy distance matrix $\hat{\mathbf{D}}$.

Output: A cleaned distance matrix $\mathbf{D}$.

Procedure:

1. Set $i=0$ and $\hat{\mathbf{Q}}^{(i)}=\varphi_{1}(\hat{\mathbf{Q}})$, where $\hat{\mathbf{Q}}$ is obtained by squaring every element of $\hat{\mathbf{D}}$.

2. Compute $\hat{\mathbf{Q}}_{2}^{(i)}=\varphi_{2}\left(\hat{\mathbf{Q}}^{(i)}\right), \hat{\mathbf{Q}}_{3}^{(i)}=\varphi_{3}\left(\hat{\mathbf{Q}}_{2}^{(i)}\right)$, and $\hat{\mathbf{Q}}^{(i+1)}=\varphi_{4}\left(\hat{\mathbf{Q}}_{3}^{(i)}\right)$.

3. If $\left\|\hat{\mathbf{Q}}^{(i+1)}-\hat{\mathbf{Q}}^{(i)}\right\|<\epsilon$ for some prescribed threshold $\epsilon$, go to step 4 . Otherwise, set $i \leftarrow i+1$ and go to step 2 .

4. Output the matrix $\mathbf{D}$ obtained by taking the square root of every element of $\hat{\mathbf{Q}}^{(i+i)}$.

beginning of the algorithm. The convergence of this iterative procedure to a matrix that exhibits all of the four desired properties is supported by the following result.

Proposition 1. Any matrix sequence generated using Algorithm 2 contains a sub-sequence which converges to a matrix satisfying the four desired properties.

Proof. The functions $\varphi_{2}$ and $\varphi_{3}$ are continuous point-to-point mappings, and $\varphi_{4}$ is a closed point-to-set mapping (Theorem 2 of Ref. 11). Therefore, the composite point-to-set mapping $\varphi=\varphi_{4} \varphi_{3} \varphi_{2}$ used in Algorithm 2 is closed (Lemma 2 of Ref. 11). From Theorem 1 of Ref. 11, any sequence generated according to the update rule $\hat{\mathbf{Q}}^{(i+1)} \in \varphi\left(\hat{\mathbf{Q}}^{(i)}\right)$ thus contains a subsequence that converges to a matrix satisfying the desired properties. $\square$

It can be easily checked that the Frobenius norm of the distance matrix is reduced at each iteration. This reduction quantifies the amount of noise that is removed by the method. In practice, we have observed that most of the noise can be removed in less that 10 iterations. Note that additional constraints can be enforced on the matrix $\mathbf{Q}$ depending on the setup under consideration. For example, if the transducers are uniformly spaced on a ring of unknown radius, the matrix $\mathbf{Q}$ has a circular form and its rank is no greater than 3 . In this case, circularity can be enforced by averaging the coefficients whose coordinates $(i, j)$ satisfy $|i-j|=k$ for some $k \in\{0,1, \ldots, n-1\}$.

In our implementation, Algorithm 2 is used to denoise the distance estimates (8) prior to the computation of the positions using the MDS algorithm. The proposed method is summarized in Algorithm 3. It can be viewed as an extension of the technique proposed in Ref. 8 with an additional iterative denoising procedure. Note that the estimated propagation speed and the distance matrix obtained from Algorithm 2 allow us to compute denoised time of flight data. This is useful, for example, to compute reference time of flights for a reconstruction algorithm based on travel time tomography. ${ }^{13}$ Let us now assess the accuracy of the proposed method both in simulated and experimental scenarios.

\section{RESULTS}

\subsection{Simulations}

We first consider a synthetic transducer array made of $n=256$ transducers whose positions are only approximatively spaced on a ring of radius $r=100 \mathrm{~mm}$ with a uniform angular spacing. More specifically, we consider the position estimates obtained in the experimental setup described in Section 5.2. Measurements are synthesized using the relationships (1) and (2) with a propagation speed of $c=1500 \mathrm{~m} / \mathrm{s}$. We chose fixed emission and reception delays according to the following sinusoidal profiles

$$
e_{i}=\tau+\frac{\tau}{4} \sin \left(\frac{2 \pi i}{n}\right) \quad \text { and } \quad r_{i}=\tau+\frac{\tau}{4} \sin \left(\frac{2 \pi i}{n}+\pi\right),
$$


Algorithm 3 Calibration

Input: A (possibly incomplete) measurement vector $\hat{\mathbf{m}}$ and an initial distance vector $\hat{\mathbf{d}}_{0}$.

Output: Position estimates $\hat{\mathbf{X}}$, propagation speed estimate $\hat{c}$, and delay estimates $\hat{\mathbf{e}}$ and $\hat{\mathbf{r}}$.

Procedure:

1. Compute $\hat{c}$, $\hat{\mathbf{e}}$ and $\hat{\mathbf{r}}$ from (9) using $\hat{\mathbf{m}}$ and $\hat{\mathbf{d}}_{0}$.

2. Interpolate the missing measurements using $\hat{c}, \hat{\mathbf{e}}$ and $\hat{\mathbf{r}}$ in (1) and (2).

3. Update the distance estimates from (8).

4. Denoise the distance estimates using Algorithm 2.

5. Compute the position estimates $\hat{\mathbf{X}}$ with the MDS algorithm using the denoised distance estimates.

6. Output $\hat{\mathbf{X}}, \hat{c}, \hat{\mathbf{e}}$ and $\hat{\mathbf{r}}$.

where $\tau=0.2 \mu \mathrm{s}$. Additive white Gaussian noise is added to the measurements to meet a prescribed signal-tonoise ratio (SNR). The initial distance estimates used in Algorithm 3 are obtained from a uniform spacing on a ring of radius $r$. Results are averaged over 100 realizations.

In Figure 2(a), we first compare the reconstruction accuracy of the positions with and without the distance matrix denoising method described in Algorithm 2. We also compare the accuracy of the proposed method to the direct minimization of (5) using the trust region optimization method lsqonlin in Matlab with the default optimization parameters. To this end, we evaluate the root mean square error (RMSE) of the corresponding distance matrices. It is given by

$$
\operatorname{RMSE}=\sqrt{\frac{1}{n^{2}} \mathcal{E}\left\{\|\mathbf{D}-\hat{\mathbf{D}}\|^{2}\right\}}
$$

where $\mathcal{E}$ denotes expectation. We clearly observe that the proposed iterative denoising technique allows us to reduce the error in the position estimates and to obtain an accuracy that is slightly worse than the full optimization (5) in the low SNR regime, but slightly better in the high SNR regime.

To evaluate the sensitivity of the proposed method to the initial position estimates, we plot in Figure 2(b) the error curves obtained from a uniform ring geometry with radii 100, 120 and $140 \mathrm{~mm}$. In this example, we note that the calibration error increases smoothly as the initial radius departs from the true average distance between the transducers and the center of the array. A similar degradation is observed when solving the original optimization problem (5).

In Figure 2(c), we compare the computation time required by the proposed method and the full minimization (5) as a function of the number of transducers. We observe that the proposed method provides similar reconstruction accuracy but has a much lower computational complexity. The proposed technique is thus more suitable, for example, in a sensor network scenario where calibration must be done by devices with only limited power available.

\subsection{Application to Ultrasound Transducers}

We now apply the proposed method to the calibration of a set of $n=256$ ultrasound transducers used in a clinical prototype for breast cancer detection at the Karmanos Cancer Institute. ${ }^{3}$ The transducers are assumed to be uniformly spaced on a ring of radius $r=99.9 \mathrm{~mm}$. Furthermore, the overall emission/reception delay of each transducer pair is assumed to be equal to a constant $\tau$. This amounts to setting the matrices $\mathbf{K}_{e}$ and $\mathbf{K}_{r}$ in (7) as the all-one vector and choosing $\mathbf{e}$ and $\mathbf{r}$ as the scalar $\tau / 2$. Time delays are computed from signals measured in water, hereafter referred to as a water shot. A time delay estimation method based on the arrival of the first peak of the transmitted signal is used in Ref. 13. The obtained measurement matrix is depicted in Figure 3. Due to the directional response of the transducers, the signals transmitted between adjacent pairs of emitters and receivers have a low signal-to-noise ratio and the corresponding time delays cannot be computed 


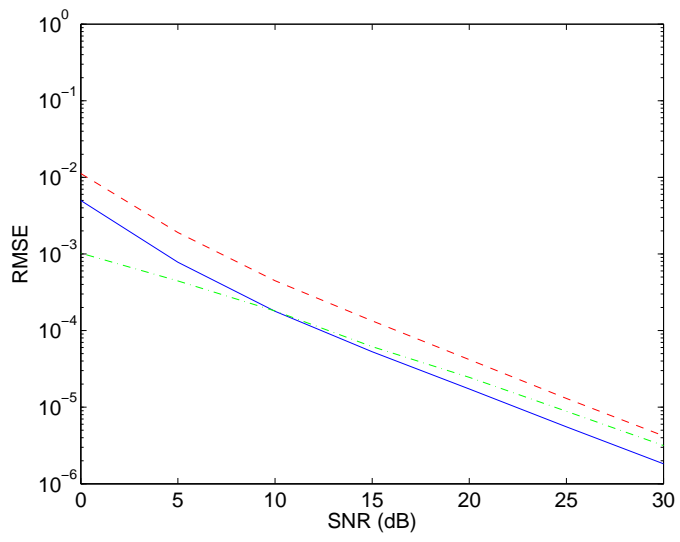

(a)

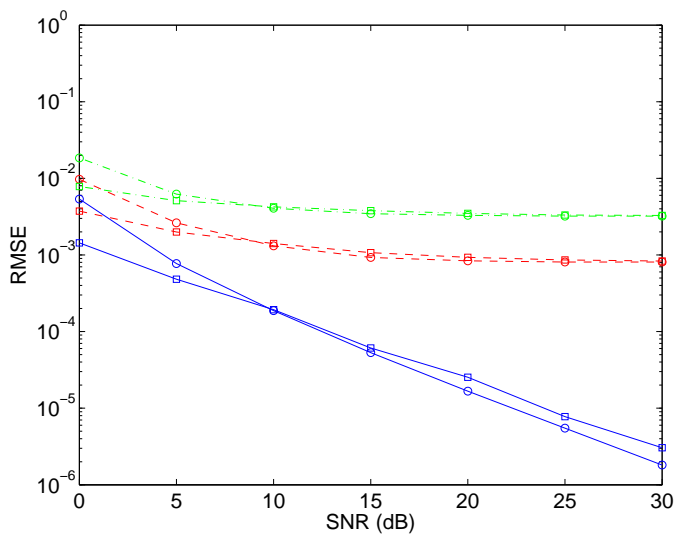

(b)

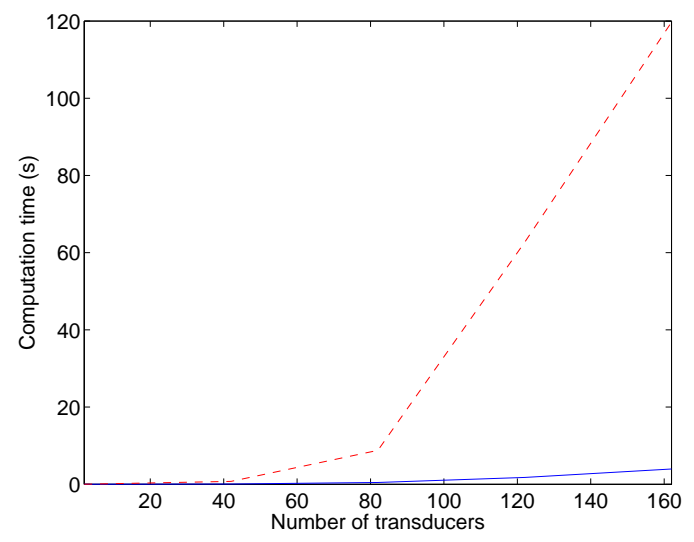

(c)

Figure 2. Calibration results. (a) Estimation error of the transducer positions as a function of the input SNR with (solid) and without (dashed) the proposed denoising technique. The error curve obtained by directly minimizing (5) is also plotted (dash-dotted). (b) Estimation error of the transducer positions as a function of the input SNR using a uniform ring geometry with radius $100 \mathrm{~mm}$ (solid), $120 \mathrm{~mm}$ (dashed) and $140 \mathrm{~mm}$ (dash-dotted) as initial estimate. The proposed method is labeled with circles, the result of the minimization (5) is labeled with squares. (c) Computation time of the proposed method (solid) and the minimization (5) (dashed) as a function of the number of transducers.

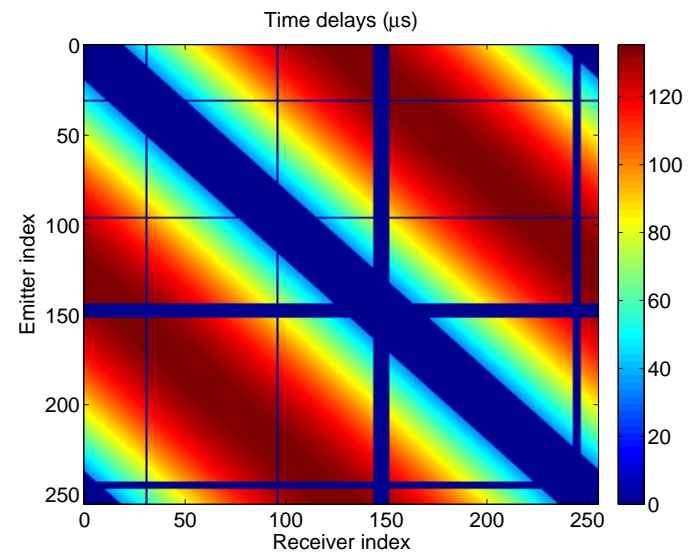

Figure 3. Measurement matrix computed from a water shot. 


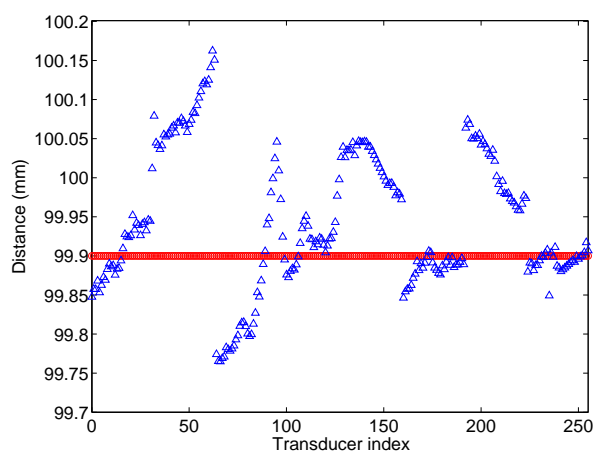

(a)

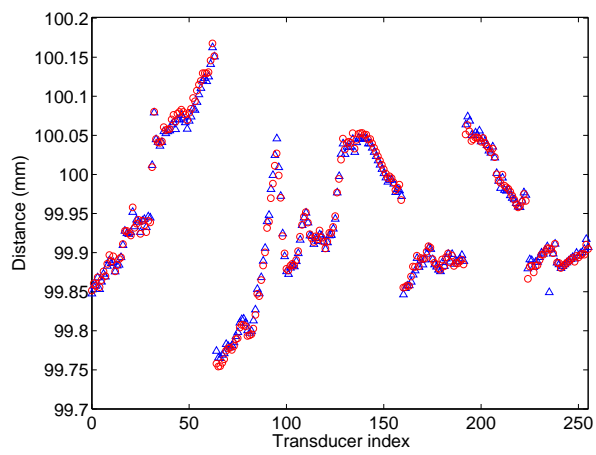

(c)

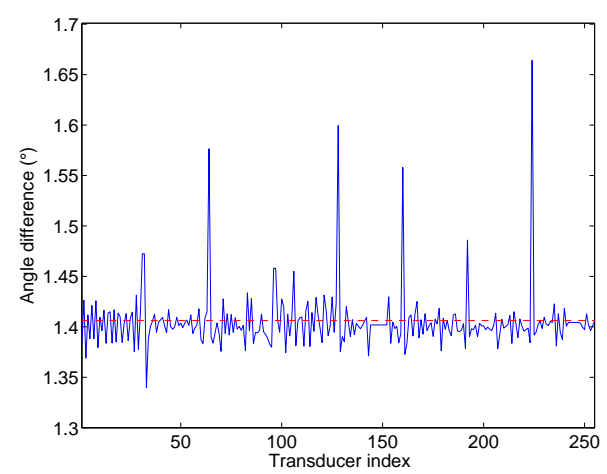

(b)

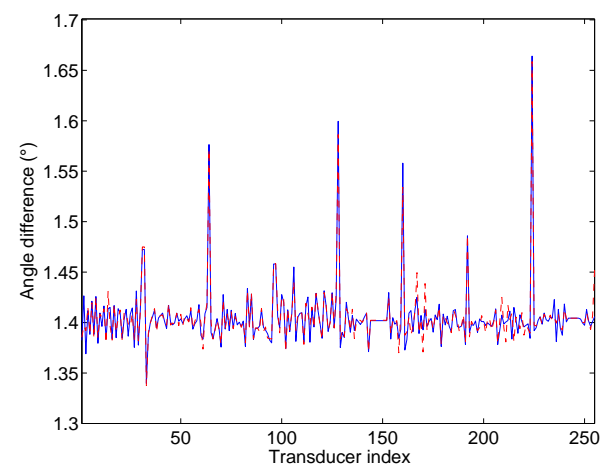

(d)

Figure 4. Transducer positions. (a) Distance of each transducer to the center of the array for the estimated (triangles) and assumed (circles) positions. (b) Angle difference between consecutive transducers for the estimated (solid) and assumed (dashed) positions. (c) Distance to the center of the array for the first (triangles) and second (circles) estimated positions. (d) Angle difference between consecutive transducers for the first (solid) and second (dashed) estimated positions.

reliably. In our case, the 20 first off-diagonal elements of the measurement matrix are missing. Moreover, the transducers 30, 96, 144 to 151, and 243 to 246 are considered as too noisy and are discarded.

The algorithm was run on the above time delay data. Figures 4(a) and 4(b) show how the estimated positions differ from the assumed uniform ring geometry. In Figure 4(a), we plot the distance of every element to the center of the array. We clearly see how the values depart from the assumed constant radius $r$. The method is able to resolve displacements of the order of $0.01 \mathrm{~mm}$. We also observe a block structure which reflects the way the array was built, that is, by concatenating 8 small curved arrays of 32 transducers each. In Figure 4(b), we plot the angle differences between two consecutive transducers and compare it to the uniform spacing of the assumed ring geometry. We observe significant differences every 32 elements, corresponding to consecutive transducers lying on two different curved arrays. Note that the positions of the bad transducers have been linearly interpolated (in radius and angle) between the closest good transducers. For this water shot, the estimated sound speed is $\hat{c}=1509.6 \mathrm{~m} / \mathrm{s}$, and the delay of the transducers, including the systematic delay introduced by the time delay estimation method (i.e., the time to the first peak), is about $2.68 \mu \mathrm{s}$. The temperature of the water during the experiment was also measured using a thermometer. It is given by $T=30.374{ }^{\circ} \mathrm{C}$ which translates into a sound speed of $c=1510.1 \mathrm{~m} / \mathrm{s}$ according to the formula ${ }^{16}$

$$
c=1405.03+4.624 T-0.0383 T^{2} .
$$

Note that the above formula is accurate for temperatures between 10 and $40{ }^{\circ} \mathrm{C}$. The water sound speed is thus estimated with high accuracy. To check the consistency of the method, the calibration was performed using another set of measurements corresponding to a different water temperature. In this case, the estimated sound speed is $\hat{c}=1500.7 \mathrm{~m} / \mathrm{s}$, and the delay is about $2.64 \mu \mathrm{s}$. The temperature of the water during the experiment is 


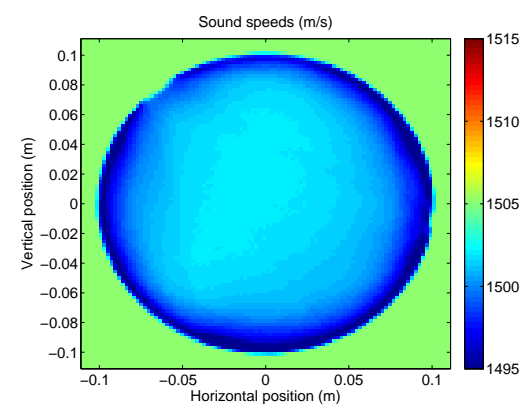

(a)

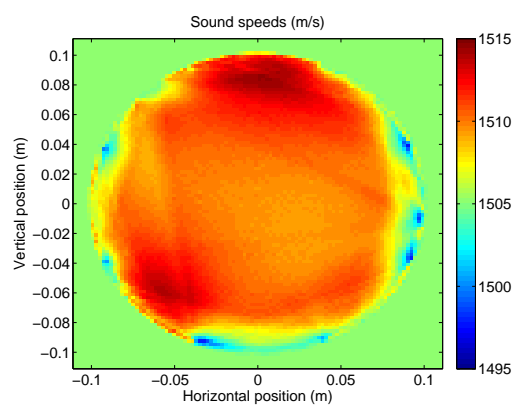

(b)

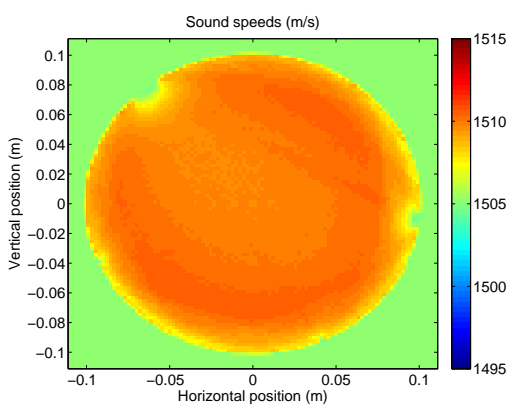

(c)

Figure 5. Sound speed reconstruction of a water shot using travel time tomography. (a) Non-calibrated setup. Calibrated delays but non-calibrated positions. (c) Calibrated delays and positions.

$T=26.6{ }^{\circ} \mathrm{C}$ which corresponds to a sound speed of $c=1500.9 \mathrm{~m} / \mathrm{s}$ according to (10). In Figures $4(\mathrm{c})$ and $4(\mathrm{~d})$, we plot the estimates of the positions of the transducers using the two different water shots. We clearly observe a good consistency which suggests that the calibration parameters are well estimated.

Finally, we illustrate, in Figure 5, the effect of calibration on the reconstruction of the sound speeds in water. To this end, we use the travel time tomography reconstruction method described in Ref. 13, with an initial uniform sound speed estimate of $1505 \mathrm{~m} / \mathrm{s}$. In Figure 5(a), we plot the reconstruction obtained with the noncalibrated setup. We observe a large ring artifact of low sound speeds around the array of transducers. Moreover, the sound speed is not properly estimated, even in the center of the array. This is due to the fact that the delays of the transducers are not well compensated for. In Figure 5(b), we account for these delays but keep the noncalibrated positions. We can see that the low sound speed region disappears but that important inhomogeneities remain. The reconstruction obtained with the calibrated setup is plotted in Figure 5(c). We observe that many of the remaining artifacts vanish and that the uniform sound speed is more accurately estimated. Note that, in these three scenarios, no processing was performed on the original data as a means to denoise it. In fact, our goal is only to demonstrate the effect of a more accurate calibration. Hence, a number of reconstruction artifacts remain, in particular at the boundary of the ring where only few measurements are available. We expect the benefit of the proposed calibration method to be even more important using a waveform tomography inversion scheme, typically more accurate but much less robust to model mismatch. ${ }^{14}$

\section{CONCLUSIONS}

We investigated a calibration problem for an array of transducers in an unknown homogeneous medium. A technique was proposed to estimate the positions and the internal delays of the transducers, as well as the speed of propagation in the medium. Robustness of the method to noise and model mismatch has been addressed by means of a novel iterative technique that denoises the distance matrix involved in the optimization process. The convergence of this procedure has been proved. Finally, the effectiveness of our approach has been demonstrated both in simulated scenarios and with experimental data obtained from an ultrasound scanner designed for breast cancer detection. ${ }^{3}$ 


\section{REFERENCES}

1. S. Doclo and M. Moonen, "Multi-microphone noise reduction using recursive GSVD-based optimal filtering with ANC postprocessing stage," IEEE Transactions on Speech and Audio Processing 13, pp. 53-69, Jan. 2005.

2. D. F. Nadeau, W. Brutsaert, M. Parlange, E. Bou-Zeid, G. Barrenetxea, O. Couach, M.-O. Boldi, J. S. Selker, and M. Vetterli, "Estimation of urban sensible heat flux using a dense wireless network of observations," Environmental Fluid Mechanics 9, pp. 635-653, Sept. 2009.

3. N. Duric, P. Littrup, L. Poulo, and A. Babkin, "Detection of breast cancer with ultrasound tomography: First results with the Computed Ultrasound Risk Evaluation (CURE) prototype.," Medical Physics 34, pp. 773-785, Feb. 2007.

4. Y. Li, "Position and time-delay calibration of transducer elements in a sparse array for underwater ultrasound imaging," IEEE Transactions on Ultrasonics, Ferroelectrics, and Frequency Control 53, pp. 1458-1467, Aug. 2006.

5. R. C. Waag and R. J. Fedewa, "A ring transducer system for medical ultrasound research," IEEE Transactions on Ultrasonics, Ferroelectrics, and Frequency Control 53, pp. 1707-1718, Oct. 2006.

6. C. H. Knapp and G. C. Carter, "The generalized correlation method for estimation of time delay," IEEE Transactions on Acoustics, Speech and Signal Processing ASSP-24, pp. 320-327, Apr. 1976.

7. Y. T. Chan, J. M. Riley, and J. B. Plant, "A parameter estimation approach to time-delay estimation and signal detection," IEEE Transactions on Acoustics, Speech and Signal Processing 28, pp. 8-16, Feb. 1980.

8. P. Drineas, A. Javed, M. Magdon-Ismail, G. Pandurangant, R. Virrankoski, and A. Savvides, "Distance matrix reconstruction from incomplete distance information for sensor network localization," in 3rd Annual IEEE Communications Society Conference on Sensor, Mesh and Ad Hoc Communications and Networks, pp. 536-544, Sept. 2006.

9. G. Mao, B. Fidan, and B. Anderson, "Wireless sensor network localization techniques," Computer Networks 51, pp. 2529-2553, 2007.

10. A. Ihler, J. Fisher, R. Moses, and A. Willsky, "Nonparametric belief propagation for self-localization of sensor networks," IEEE Journal on Selected Areas in Communications 23(4), pp. 809-819, 2005.

11. J. A. Cadzow, "Signal enhancement - A composite property mapping algorithm," IEEE Transactions on Acoustics, Speech and Signal Processing 36, pp. 49-62, Jan. 1988.

12. M. Fazel, H. Hindi, and S. P. Boyd, "Log-det heuristic for matrix rank minimization with applications to hankel and euclidean distance matrices," in Amer. Control Conf., 3, pp. 2156-2162, June 2003.

13. I. Jovanovic, Inverse Problems in Acoustic Tomography: Theory and Applications. PhD thesis, EPFL, Lausanne, Switzerland, 2008.

14. R. G. Pratt, "Seismic waveform inversion in the frequency domain, part 1: Theory, and verification in a physical scale model," Geophysics 64, pp. 888-901, May-June 1999.

15. K. Y. Foo and P. R. Atkin, "A relative-localization algorithm using incomplete pairwise distance measurements for underwater applications," EURASIP Journal on Advances in Signal Processing 2010, 2010.

16. R. Graaff and J. Lubbers, "A simple and accurate formula for the sound velocity in water.," Ultrasound in Medicine and Biology 24, pp. 1065-1068, July 1998. 